\title{
Nonuniversal Quasi-Long Range Order in the Glassy Phase of Impure Superconductors
}

\author{
Thorsten Emig, Simon Bogner and Thomas Nattermann \\ Institut für Theoretische Physik, Universität zu Köln, Zülpicher Str. 77, D-50937 Köln, Germany
}

(June 5, 2018)

\begin{abstract}
The structural correlation functions of a weakly disordered Abrikosov lattice are calculated for the first time in a systematic RG-expansion. In the asymptotic limit the Abrikosov lattice exhibits still quasi long range translational order described by a nonuniversal exponent $\bar{\eta}_{\mathbf{G}}$ which depends on the ratio of the renormalized elastic constants $\kappa=\tilde{c}_{66} / \tilde{c}_{11}$ of the flux line (FL) lattice. Our calculations show clearly three distinct scaling regimes corresponding to the Larkin, the manifold and the asymptotic Bragg glass regime. The manifold roughness exponent $\zeta_{\mathrm{rm}}(\kappa)$ is also nonuniversal. Our results are at variance with those of the variational treatment with replica symmetry breaking which allows in principle an experimental discrimination between the two approaches.
\end{abstract}

PACS numbers: 74.60.Ge, 05.20.-y

Conventional type-II superconductors show in addition to the flux repulsing Meissner state a second superconducting (Abrikosov) phase in which the magnetic induction $\mathbf{B}$ enters the material in the form of quantized flux lines (FLs) which form a triangular lattice. Each FL carries a unit flux quantum $\phi_{0}=h c / 2 e$. The Abrikosov lattice is characterized by a non-zero shear modulus $c_{66}$, which vanishes at the upper and lower critical fields, $H_{c_{2}}$ and $H_{c_{1}}$, where continuous transitions to the normal and the Meissner state, respectively, occur. Abrikosov in his mean-field solution treats FLs as stiff rods.

Thermal fluctuations roughen the FLs resulting in a melting of the Abrikosov lattice close to $H_{c_{1}}$ and $H_{c_{2}}$, respectively, because of the softening of $c_{66}$. This applies in particular to high- $T_{c}$ materials with their elevated transition temperatures and their pronounced layer structures [1]. At present, it is not clear, whether the transition to the normal phase at high field happens in these materials via one or two transitions. However, melting of the FL lattice has clearly been observed experimentally [2,3].

It is well known, that in addition to thermal fluctuations in type-II superconductors the effect of disorder has to be taken into account since FLs have to be pinned in order to prevent dissipation from their motion under the influence of an external current. Randomly distributed pinning centers lead indeed to a destruction of the Abrikosov lattice, as has been first demonstrated by Larkin using perturbation theory th. In particular, he found an exponential decay of the correlations of the order parameter for translational long range order $\Psi_{\mathbf{G}}(\mathbf{x})=\mathbf{e}^{\mathbf{i G u ( x )}}$ on length scales larger than a disorder dependent Larkin length $L_{\xi}$. Here $\mathbf{G}$ and $\mathbf{u}$ denote a reciprocal lattice vectors and the displacement field of the FL lattice, respectively, and $\mathbf{x}=\left(\mathbf{x}_{\perp}, \mathbf{z}\right)$ is a $\mathrm{d}$ dimensional position vector.

However, as was first shown by Nattermann [5], in treating the interaction between the FL lattice and the disorder, it is crucial to keep the periodicity of this inter- action under the transformation $\mathbf{u} \rightarrow \mathbf{u}+\mathbf{R}$, where $\mathbf{R}$ is a vector of the Abrikosov lattice. This symmetry, which is abandoned in perturbation theory [4] and in the so-called manifold models [6], leads to a much slower, logarithmic increase of the elastic distortions with the system size $L$ [5, 7, 8]. This results in a power law decay of the pair correlation function $C_{\mathbf{G}}(\mathbf{x})=\left\langle\Psi_{\mathbf{G}}(\mathbf{x}) \Psi_{-\mathbf{G}}(\mathbf{0})\right\rangle$. In particular, Giamarchi and Le Doussal [8] calculated $C_{\mathbf{G}}(\mathbf{x})$ using (i) a variational treatment for the triangular FL lattice and (ii) a functional renormalization group (FRG) for a simplified model using a scalar displacement field $u$ only. In both cases they found $C_{\mathbf{G}}\left(\mathbf{x}_{\perp}, 0\right) \sim\left|\mathbf{x}_{\perp}\right|^{-\bar{\eta}_{\mathbf{G}}}$ with $\bar{\eta}_{\mathbf{G}_{\mathbf{0}}}=A(4-d)$ where $d$ denotes the space dimensionality. $\quad \mathbf{G}_{0}$ is one of the smallest reciprocal lattice with $G_{0} a=4 \pi / \sqrt{3}, a=\left(2 \phi_{0} / \sqrt{3} B\right)^{1 / 2}$ denotes the lattice spacing and $A$ is a universal constant $(A=1$ and $A=\pi^{2} / 9 \approx 1.1$ for the treatment (i) and (ii), respectively). Thus, even with (weak) disorder there is a quasilong range ordered flux phase which shows Bragg peaks, the "Bragg-glass" [8]. This result is believed to be valid in an extended region of the $H-T$ phase diagram up to a phase boundary, where the occurrence of unbounded dislocation loops - ignored so far - leads to an instability of the Bragg glass phase [9 14].

The power law decay of $C_{\mathbf{G}}(\mathbf{x})$ is reminiscent of the situation in pure $2 D$-crystals where in the solid phase $C_{\mathbf{G}}\left(\mathbf{x}_{\perp}\right) \sim\left|\mathbf{x}_{\perp}\right|^{-\eta_{\mathbf{G}}}$. This solid phase is indeed a line of critical points with $\eta_{\mathbf{G}}=T G^{2}\left(1+\kappa^{-1}\right) /\left(4 \pi \tilde{c}_{11}\right)$ and $\kappa=\tilde{c}_{66} / \tilde{c}_{11}$. The $\tilde{c}_{i i}$ represent the renormalized elastic constants which have a finite temperature dependent value. At the melting temperature $T_{m} \eta_{\mathbf{G}_{0}}=\frac{1}{3}\left(1-\kappa^{2}\right)$. [15]. Similarly, the $2 D$ disordered crystal close to the glass temperature $T_{g}$ (i.e. $\eta_{\mathbf{G}_{\mathbf{0}}} \lesssim 2$ ) is described by a line of fixed points which depends on $\kappa$ 16.

On the contrary, the Bragg glass phase is characterized by a zero temperature fixed point where the ratio between the disorder strength and the elastic energy vanishes on large scales $L$ as $\Delta^{*} L^{d-4}$. It has been suggested in [8], 
that the fixed point value $\Delta^{*}$ might be universal resulting in a universal coefficient $A$ of $\bar{\eta}_{\mathbf{G}}$. In the present treatment we will show however, that a FRG treatment of the full triangular model leads indeed to a nonuniversal $\kappa$-dependent value of $\bar{\eta}_{\mathbf{G}}=\Delta^{*}(\kappa)(G a)^{2}$. The situation is therefore qualitatively similar to that of $2 D$ crystals at the melting temperature. Quantitatively, the effect reflects the contributions from the interaction between different Fourier modes, which are not considered in the variational treatment [7,8]. With $\kappa$ dependent in general on $\mathbf{B}$ and $T$, the observation of a field-dependent $\bar{\eta}_{\mathbf{G}}$ would yield the opportunity to judge the validity of different approximation schemes under debate 17].

Since in the Bragg-glass phase dislocations in the vortex lattice can be neglected [9 14], its configurations are described completely by the elastic displacement field $\mathbf{u}(\mathbf{x})$. The vortex density is then given by $\rho(\mathbf{x}, \mathbf{u})=$ $\sum_{\mathbf{R}} \delta\left(\mathbf{x}_{\perp}-\mathbf{R}-\mathbf{u}(\mathbf{R}, z)\right)$. The impurities are modeled by a Gaussian random potential $U(\mathbf{x})$ with two-point correlation $\overline{U(\mathbf{x}) U(\mathbf{0})}=\gamma \xi^{2} g\left(x_{\perp} / \xi\right) \delta(z)$. Here $\gamma=f_{\text {pin }}^{2} n_{i} \xi^{2}$, $f_{\text {pin }}$ denotes the individual pinning force, $n_{i}$ the impurity density, $\xi$ the maximum of the coherence and disorder correlation length [1] and $g$ is a short ranged function. On scales larger than the London penetration depth $\lambda$ the FL lattice can be described by local elasticity theory, leading to the Hamiltonian

$$
\begin{aligned}
\mathcal{H} & =\frac{1}{2} \int d^{d-2} \mathbf{z} d^{2} \mathbf{x}_{\perp}\left\{c_{11}\left(\boldsymbol{\nabla}_{\perp} \cdot \mathbf{u}\right)^{2}+c_{66}\left(\boldsymbol{\nabla}_{\perp} \times \mathbf{u}\right)^{2}+\right. \\
& \left.+c_{44}\left(\boldsymbol{\nabla}_{\mathbf{z}} \mathbf{u}\right)^{2}\right\}+\int d^{d} \mathbf{x} E_{\text {pin }}(\mathbf{u}, \mathbf{x}),
\end{aligned}
$$

where $\mathbf{x}_{\perp}=(x, y)$, and the $\mathbf{z}$ component has been generalized to a $d-2$-dimensional coordinate to allow for an $\epsilon$-expansion about 4 dimensions. The random potential is defined as $E_{\text {pin }}(\mathbf{u}, \mathbf{x})=U(\mathbf{x}) \rho(\mathbf{x}, \mathbf{u})$. The Hamiltonian is invariant under simultaneous mapping of shear onto compressional modes (rotation of $\mathbf{u}$ by $90^{\circ}$ ) and permutation of the corresponding moduli $\left(\kappa \rightarrow \kappa^{-1}\right)$. For $E_{\text {pin }}(\mathbf{u}, \mathbf{x})$ this invariance holds in a statistical sense. Thus the partition function and all thermodynamic quantities also show this symmetry. We therefore can restrict the calculations to $0 \leq \kappa \leq 1$ in the following. Results for $\kappa>1$ correspond to those for $\kappa^{-1}$ with $\mathbf{u}$ rotated.

Performing the disorder average and discarding spatially rapidly oscillating terms [8], one obtains for the disorder correlator, which is defined by $\overline{E_{\text {pin }}(\mathbf{u}, \mathbf{x}) E_{\text {pin }}(\mathbf{0}, \mathbf{0})}=R(\mathbf{u}) \delta(\mathbf{x})$, a periodic function with the Fourier representation

$$
R(\mathbf{u})=\gamma \xi^{2} \frac{B^{2}}{\phi_{0}^{2}} \sum_{\mathbf{G}} \hat{g}(G \xi) \cos (\mathbf{G u}),
$$

where $B / \phi_{0}$ is the mean area density of vortices and $\hat{g}(G \xi)$ is the Fourier transform of $g\left(x_{\perp} / \xi\right)$.

To obtain the disorder averaged configuration of the FL lattice on a particular length scale, one has to take into account the renormalization of $R(\mathbf{u})$ by fluctuations on shorter length scales. This can be done systematically by a functional renormalization group (FRG) for the replica Hamiltonian resulting from Eq. (11) after the disorder average. Because of the statistical invariance of the the replica Hamiltonian with the inter-replica coupling (2) under a shift of $\mathbf{u}$ by an arbitrary vector field inducing a compression, shear and/or tilt of the FL lattice, there is no renormalization of the elastic moduli [18]. Therefore the temperature obeys the exact flow equation $d T / d l=(d-2) T$ leading to a $T=0$ fixed point for $d>2$. Notice however that in the original model of (1) the statistical invariance is not exactly fulfilled on length scales smaller than $a$, leading to a small renormalization $c_{i i} \rightarrow \tilde{c}_{i i}$ of the elastic constants which will be considered from now on as effective parameters. In our FRG only coordinates are rescaled as $\mathbf{x} \rightarrow \exp (d l) \mathbf{x}$ to keep the cutoff $\Lambda$ fixed with $d l$ the infinitesimal width of the momentum shell. Because of the dispersion of the elastic constants on scales smaller than the penetration depth $\lambda$, we have to choose here $\Lambda \approx 2 \pi / \lambda$. Fluctuations on smaller scales can be ignored if the Larkin length $L_{\xi}$ (see below) is much larger then $\lambda$, i.e. for weak disorder. For larger disorder one has to take into account the dispersion $\tilde{c}_{11}$ and $\tilde{c}_{44}$ which will result in a more complicated cross-over but not affect the asymptotic behavior. The flow equation for $R(\mathbf{u})$ can then be derived along the lines of Refs. 19,20. Contrary to previous cases, after suitable rescaling of $R(\mathbf{u})$, we obtain

$$
\begin{gathered}
\frac{d R(\mathbf{u})}{d l}=\epsilon R(\mathbf{u})+\frac{a^{2}}{2}\left\{\left(\partial_{x}^{2} R\right)^{2}+\left(\partial_{y}^{2} R\right)^{2}+2\left(\partial_{x} \partial_{y} R\right)^{2}\right. \\
\left.+2 \Delta\left(\partial_{x}^{2}+\partial_{y}^{2}\right) R-\frac{\delta}{4}\left[\left(\partial_{x}^{2} R-\partial_{y}^{2} R\right)^{2}+4\left(\partial_{x} \partial_{y} R\right)^{2}\right]\right\} \quad(3)
\end{gathered}
$$

with the dimensionless parameter $\Delta=-\partial_{x} \partial_{x} R(\mathbf{0}) \equiv$ $-\partial_{y} \partial_{y} R(\mathbf{0})$ and $\partial_{x}=\partial / \partial u_{x}$ etc.. The last equality as well as $\partial_{x} \partial_{y} R(\mathbf{0})=0$ follow from the requirement of hexagonal symmetry for $R(\mathbf{u})$. Performing the momentum shell integrals to lowest order in $\epsilon$, one obtains the anisotropy parameter

$$
\delta=1-\frac{2 \ln (\kappa)}{\kappa-\kappa^{-1}}
$$

i.e., $0 \leq \delta \leq 1$ for any ratio $\kappa=\tilde{c}_{66} / \tilde{c}_{11}$. In the isotropic case $\tilde{c}_{11}=\tilde{c}_{66}(\delta=0)$ the flow Eq. (3) reduces to that of Ref. [20], if $\tilde{c}_{11} \rightarrow \infty$, as often assumed for FL lattices, we have to put $\delta=1$.

From Eq. (2) we find that the bare, unrenormalized dimensionless parameter $\Delta_{0}$ is given by

$$
\Delta_{0} \approx 10^{-3} \Lambda^{d-4} \gamma \frac{1+\kappa}{\tilde{c}_{44} \tilde{c}_{66}} \frac{B^{2}}{\phi_{0}^{2}}(1+\mathcal{O}(\xi / a)),
$$

where we have assumed $\hat{g}(G \xi)=\xi^{2} \Theta(1-G \xi)$ with $\Theta$ the step function and $\xi \ll a$ to evaluate the sum over $\mathbf{G}$. The Larkin length (parallel to $\mathbf{B}$ ) in three dimensions is given by $L_{\xi} \approx \xi^{2} / \Delta_{0} \Lambda a^{2}$ [1]. The condition of weak disorder mentioned above reads then $\Delta_{0} a^{2} \ll \xi^{2}$.

Now we integrate Eq. (3) to obtain the renormalized function $R(\mathbf{u})$ on all length scales $L=\exp (l) / \Lambda$ including the fixed point $R^{*}(\mathbf{u})$ for $L \rightarrow \infty$. With the bare corre- 


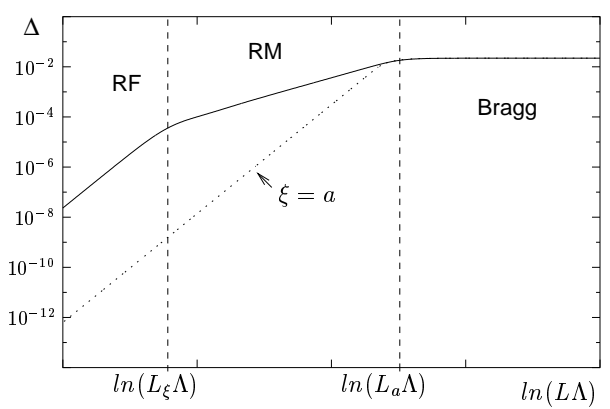

FIG. 1. RG flow of $\Delta(l)$ through three regimes obtained by numerical integration of Eq. (3).

lator of Eq. (2) showing the full symmetry of the triangular lattice - translation, 6-fold rotational axis, 3 mirror lines - and the flow of Eq. (3) preserving these symmetries as it ought to, the set of possible solutions is restricted to functions with the full lattice symmetry on every length scale. Even for $R^{*}(\mathbf{u})$ an analytic solution not being very obvious, our approach is numerical and straightforward: The Ansatz assuring translational plus inversion invariance is $R(\mathbf{u})=\sum_{\mathbf{G}} R_{\mathbf{G}} \cos (\mathbf{G u})$ with the sum running over the reciprocal lattice. An infinite set of coupled nonlinear but ordinary flow equations for the coefficients $R_{\mathrm{G}}$ is derived. Rather than solving directly for the fixed point $R_{\mathrm{G}}^{*}$, the flow equations are integrated numerically with the remaining point group symmetries exploited here. Convergence to a fixed point from a huge bassin of attraction is observed. Of special interest is the flow of $\Delta(l)$ since it determines the scale dependence of the renormalized propagator $\Delta_{\text {eff }}(q) / q^{4}$ with $\Delta_{\text {eff }}(q)=\Delta(\ln (\Lambda / q))(q / \Lambda)^{4-d}$ and thus the exponents $\bar{\eta}_{\mathbf{G}}$ and $\zeta_{r m}$. Obviously, the numerical integration has to be restricted to a finite set of $R_{\mathbf{G}}$ with $|\mathbf{G}|<\left|\mathbf{G}_{c}\right|$, but high accuracy of the flow of $\Delta(l)$ can be restored by including up to 360 different coefficients and a finite size scaling like extrapolation. The accuracy for $L \rightarrow \infty$ is controlled via the exact ( $\delta$-independent) relation $\epsilon R(\mathbf{0})=(a \Delta)^{2}$ stemming from (3).

Two different but typical kinds of behavior of $\Delta(l)$ are shown in Fig. 1. Depending on the ratio $\xi / a$, two or three scaling regimes with different roughness exponent $\zeta$ defined by $\Delta(l) \sim \exp (2 \zeta l)$ can be clearly identified. For $\xi / a \ll 1$, one finds a first crossover from the random force $(\mathrm{RF})$ regime $(\zeta=\epsilon / 2)$ to the random manifold $(\mathrm{RM})$ regime $\left(\zeta=\zeta_{\mathrm{rm}}\right)$ at the Larkin length $L_{\xi}$ where $u \simeq \xi$ and a second one at the positional correlation length $L_{a} \approx L_{\xi}(a / \xi)^{1 / \zeta_{r m}}$ where $u \simeq a$ to the Bragg glass regime $(\zeta=0)$. If $\xi / a \simeq 1, L_{a} \rightarrow L_{\xi}$ and the $\mathrm{RM}$ regime disappears. Just like in the 1-d (or CDW) case [8] a cusp in the second derivatives of $R(\mathbf{u})$ develops leading to the fixed point shape shown in Fig. 2 . The length scale $L_{\mathrm{c}}$ on which the cusp appears can be determined analytically from the divergence in the RG flow of $\partial_{x}^{4} R(\mathbf{0})=\partial_{y}^{4} R(\mathbf{0})=3 \partial_{x}^{2} \partial_{y}^{2} R(\mathbf{0})$. As can be expected, $L_{\mathrm{c}}$ is related to the Larkin length $L_{\xi}$ known from perturbation theory by a numerical factor only. But interestingly we obtain the typical ratio $L_{\mathrm{c}} / L_{\xi} \approx 100$, i.e., the cusp appears clearly beyond the Larkin scale. The nonuniversality of the exponents $\zeta_{\mathrm{rm}}(\kappa)$ and $\bar{\eta}_{\mathbf{G}}(\kappa)$ can be determined from $\Delta(l)$ via the slope in the RM regime and $\Delta^{*}$, respectively. Their dependence on $\kappa$ is presented in Fig. 3.

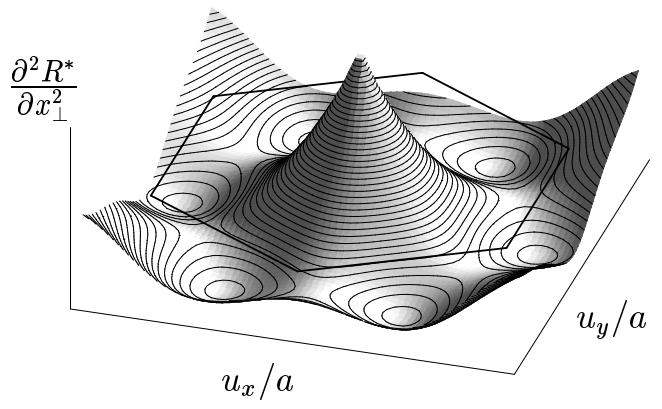

FIG. 2. Characteristic of the Bragg glass: a cusp in $\partial_{x_{\perp}}^{2} R^{*}(\mathbf{u})$ at the lattice sites.

With the numerical value for $\Delta^{*}(\kappa)$ at hands we can now calculate the displacement correlations $B_{a b}(\mathbf{x})=\overline{\left\langle\left[u_{a}(\mathbf{x})-u_{a}(\mathbf{0})\right]\left[u_{b}(\mathbf{x})-u_{b}(\mathbf{0})\right]\right\rangle}$ in the Bragg glass phase. Introducing the rescaled $z$-coordinates $z_{t}=$ $\left(\tilde{c}_{66} / \tilde{c}_{44}\right)^{1 / 2} z, z_{l}=\left(\tilde{c}_{11} / \tilde{c}_{44}\right)^{1 / 2} z$ and $h(t)=t^{-2} \ln (1+$ $\left.t^{2}\right)$, we find for $x \gg L_{a}$

$$
\begin{aligned}
& B_{x x}(\mathbf{x})=\frac{\Delta^{*}(\kappa) a^{2}}{1+\kappa}\left\{\ln \left(\frac{x_{\perp}^{2}+z_{t}^{2}}{L_{a}^{2}}\right)+\kappa \ln \left(\frac{x_{\perp}^{2}+z_{l}^{2}}{L_{a}^{2}}\right)+\frac{y^{2}-x^{2}}{x_{\perp}^{2}}\left[1-\kappa-h\left(\frac{x_{\perp}}{z_{t}}\right)+\kappa h\left(\frac{x_{\perp}}{z_{l}}\right)\right]\right\} \\
& B_{x y}(\mathbf{x})=\frac{2 \Delta^{*}(\kappa) a^{2}}{1+\kappa} \frac{x y}{x_{\perp}^{2}}\left\{\kappa-1-\kappa h\left(\frac{x_{\perp}}{z_{l}}\right)+h\left(\frac{x_{\perp}}{z_{t}}\right)\right\}
\end{aligned}
$$

and $B_{y y}(\mathbf{x})$ follows from $B_{x x}(\mathbf{x})$ by permuting $\tilde{c}_{11}$ and $\tilde{c}_{66}$. These correlations lead to the translational order correlation function $C_{\mathbf{G}}(\mathbf{x})=\overline{\langle\exp (i \mathbf{G}[\mathbf{u}(\mathbf{x})-\mathbf{u}(\mathbf{0})])\rangle}$, which reads

$C_{\mathbf{G}}(\mathbf{x}) \sim g_{\mathbf{G}} L_{a}^{\bar{\eta}_{\mathbf{G}}}\left(x_{\perp}^{2}+z_{t}^{2}\right)^{-\bar{\eta}_{\mathbf{G}} /(2(1+\kappa))}\left(x_{\perp}^{2}+z_{l}^{2}\right)^{-\bar{\eta}_{\mathbf{G}} /(2(1+1 / \kappa))}$ with $\bar{\eta}_{\mathbf{G}}=\Delta^{*}(\kappa)(a G)^{2}$ and the geometrical factor

$$
\begin{aligned}
& g_{\mathbf{G}}=\exp \left[\frac{\Delta^{*}(\kappa)(a G)^{2}}{1+\kappa}\left(\left(\hat{\mathbf{x}}_{\perp} \hat{\mathbf{G}}\right)^{2}-\frac{1}{2}\right)\right. \\
& \left.\times\left\{\left(1-h\left(\frac{x_{\perp}}{z_{t}}\right)\right)-\kappa\left(1-h\left(\frac{x_{\perp}}{z_{l}}\right)\right)\right\}\right],
\end{aligned}
$$


which describes completely the angular dependencies of the translational order. Note that the factor $g_{\mathbf{G}}$ goes to 1 in the limit $z \rightarrow \infty$. Therefore, in this limit the dependence of $C_{\mathbf{G}}(\mathbf{x})$ on the reciprocal lattice vector $\mathbf{G}$ remains only in the exponent $\bar{\eta}_{\mathbf{G}}$. Moreover, it is interesting to note that the exponents of the algebraic decay in Eq. (8) depend on the elastic moduli as soon as $z$ is finite even without taking into account the nonuniversality of the exponent $\bar{\eta}_{\mathbf{G}}$ itself. Neglecting the non-trivial renormalization of $\Delta^{*}(\kappa)$, in the case $z=0$ the above formulae reduce to those found in 8 ].

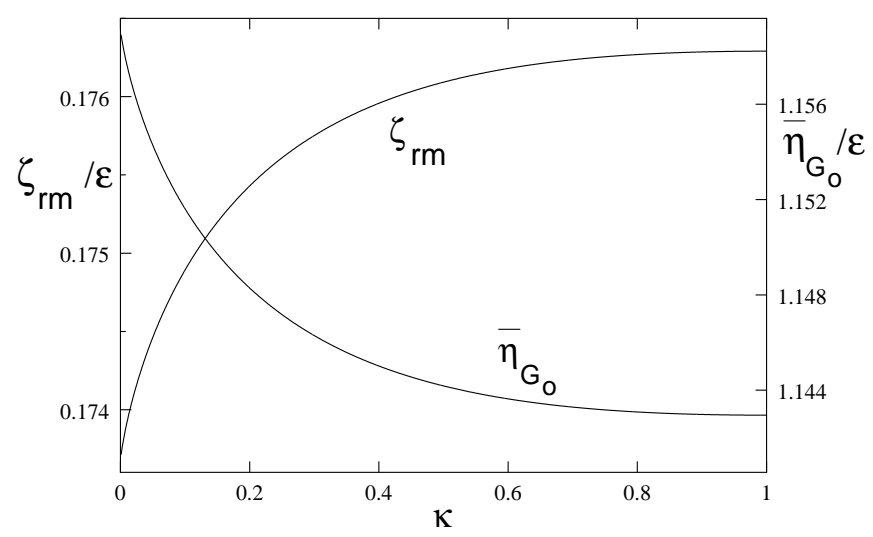

FIG. 3. Variation of the exponents $\zeta_{\text {rm }}$ and $\bar{\eta}_{\mathbf{G}_{0}}$ with $\kappa$.

The $\kappa$-dependence of $\bar{\eta}_{\mathbf{G}_{0}}$ and $\zeta_{r m}$ (Fig 3), the correlation functions $B_{a b}(\mathbf{x})$ (Eqs.(6),(7)) and $C_{\mathbf{G}}(\mathbf{x})$ (Eqs.(8),(9)) and the cross-over from the Larkin via the random manifold to the Bragg glass regime (Fig. 1) are the main results of this paper.

In isotropic superconductors at low temperatures, where flux lines interact via central forces, on has $0 \leq$ $\kappa \leq 1 / 3$. $\kappa \sim 1 / 3$ for $\lambda \leq a$, i.e. for fields close to $H_{c_{1}}$, and $\kappa \rightarrow 0$ for $H \rightarrow H_{c_{2}}$. For most of the field region $\kappa \approx \phi_{0} / 16 \pi \lambda^{2} B$, . Thus, an increase of the external field from $H_{c_{1}}$ to $H_{c_{2}}$ should result in an increase of $\bar{\eta}_{\mathbf{G}}$ and a decrease of $\zeta_{\mathrm{rm}}$. Numerically, the effect is small, since $\bar{\eta}_{\mathbf{G}_{0}}$ ranges from 1.143 to 1.159 and $\zeta_{r m}$ from 0.1737 to 0.1763 in this $\kappa$ range. Thus it will be probably hard to detect this effect. At higher temperatures, where the flux line interaction is considerable influenced by thermal interaction, as well as in anisotropic superconductors, the above inequality for $\kappa$ may not be longer fulfilled. Clearly, in the latter case also our starting Hamiltonian (1) would have to be modified.

Contact to the neutron scattering experiment is made by $S(\mathbf{G}+\mathbf{q})=\int d^{3} x e^{i \mathbf{q} \mathbf{x}} C_{\mathbf{G}}(\mathbf{x})$, the structure factor, which is proportional to the scattered intensity. For the marginal cases $\kappa=0$ and $\kappa=1$ we find from Eq. (8)

$$
S(\mathbf{G}+\mathbf{q}) \sim\left(\mathbf{q}_{\perp}^{2}+\frac{\tilde{c}_{44}}{\tilde{c}_{66}} q_{z}^{2}\right)^{\left(-3+\bar{\eta}_{\mathbf{G}}(\kappa)\right) / 2}
$$

i.e., $S(\mathbf{G}+\mathbf{q})$ exhibits Bragg peaks. For $\kappa>0$ the Fourier transform can be easily done numerically.

To summarize, we have shown that contrary to previous claims, the translational quasi-long range order in the Bragg glass phase of impure type-II superconductors is described by a nonuniversal power-like decay of the order parameter correlations. In particular, the decayexponent $\bar{\eta}_{\mathbf{G}}$ depends on the ratio $\kappa=\tilde{c}_{66} / \tilde{c}_{11}$ of the elastic constants, similar to $2 D$ pure crystals at their melting temperature. For weak disorder we find a crossover of the structural correlation functions from a Larkin-regime, where perturbation theory applies, to the random manifold regime and eventually to the asymptotic Bragg glass regime. This nonuniversality could be in principle tested by neutron scattering changing the external field.

We acknowledge discussions with H.E. Brandt, D. Feldman and S. Scheidl. This work was supported by DFG through SFB 341.

[1] G. Blatter, M. V. Feigel'man, V. B. Geshkenbein, A. I. Larkin, and V. M. Vinokur, Rev. Mod. Phys. 66, 1125 (1994).

[2] R. Cubitt et al., Nature (London) 365, 407 (1993).

[3] E. Zeldov et al., Nature (London) 375, 373 (1995).

[4] A. I. Larkin, Sov. Phys. JETP 31, 784 (1970).

[5] T. Nattermann, Phys. Rev. Lett. 64, 2454 (1990).

[6] J.-P. Bouchaud, M. Mezard, and J. S. Yedidia, Phys. Rev. Lett. 67, 3840 (1991).

[7] S. E. Korshunov, Phys. Rev. B 48, 3969 (1993).

[8] T. Giamarchi and P. Le Doussal, Phys. Rev. Lett. 72, 1530 (1994); Phys. Rev. B 52, 1242 (1995).

[9] J. Kierfeld, T. Nattermann, and T. Hwa, Phys. Rev. B 55, 626 (1997).

[10] M. Gingras and D. A. Huse, Phys. Rev. B 53, 15193 (1996).

[11] D. Carpentier, P. Le Doussal, and T. Giamarchi, Europhys. Lett. 35, 379 (1996).

[12] D. Ertaç and D. R. Nelson, Physica C 272, 79 (1996).

[13] J. Kierfeld, Physica C 300, 171 (1998).

[14] D. S. Fisher, Phys. Rev. Lett. 78, 1964 (1997).

[15] D. R. Nelson, in Phase Transitions and Critical Phenomena Vol.7, edited by C. Domb and J. L. Lebowitz, Academic Press, London, 1983.

[16] D. Carpentier and P. Le Doussal, Phys. Rev. B 55, 12128 (1997).

[17] L. Balents, J.-P. Bouchaud, and M. Mezard, J. Physique 6, 1007 (1996).

[18] T. Hwa and D. S. Fisher, Phys. Rev. Lett. 72, 2466 (1994).

[19] D. S. Fisher, Phys. Rev. Lett. 56, 416 (1986).

[20] L. Balents and D. S. Fisher, Phys. Rev. B 48, 5949 (1993). 